\title{
Magnetic Properties of Glass-Coated FeWB Microwires
}

\author{
P. Klein ${ }^{a}, *$ R. VARGA ${ }^{a}$, R. El KAmmouni ${ }^{b}$ And M. VAZQUeZ ${ }^{b}$ \\ ${ }^{a}$ Institute of Physics, Faculty of Sciences, P.J. Safarik University, Park Angelinum 9, 04154 Košice, Slovakia \\ ${ }^{b}$ Institute of Material Science of Madrid, CSIC, 28049 Madrid, Spain

\begin{abstract}
We have studied magnetization process in amorphous bistable $\mathrm{Fe}_{80} \mathrm{~W}_{3} \mathrm{~B}_{17}$ microwires with reduced Curie temperature. High mechanical stresses from glass-coating, induced during production process, result in high switching field. Reducing the length of microwire, the switching field decreases as a result of reduction of magnetoelastic anisotropy. Moreover, the decrease of magnetoelastic anisotropy results in a complex temperature dependence of the switching field. On the other hand, strong variations of the switching field with temperature can be employed in miniaturised temperature sensor.
\end{abstract}

DOI: 10.12693/APhysPolA.126.70

PACS: $75.50 . \mathrm{Kj}, 75.60 . \mathrm{Ej}$

\section{Introduction}

Amorphous glass-coated microwires are modern materials that attract attention of scientists and engineers in last decades. They consist of metallic nucleus of diameter $100 \mathrm{~nm}-50 \mu \mathrm{m}$ and glass-coating with thickness of 2-20 $\mu \mathrm{m}$. Due to their dimensions they can be employed for contactless sensing of magnetic field, temperature, mechanical stress, position etc. [1,2].

Glass-coated microwires are produced by drawing and quenching of molten master alloy together with the glass - the process that introduces strong and complex stress distribution in metallic nucleus. Their magnetization reversal process runs through the single Barkhausen jump at the field called switching field $H_{s w}$. The switching field is sensitive to external parameters like temperature or mechanical stress, that allows employment of microwires for miniature sensor applications.

One possible way to increase the sensitivity of switching field is to employ the materials with low Curie temperature [3]. Below Curie temperature, the magnetic characteristics (magnetization, magnetic anisotropy) are strongly varying.

In this report, we deal with the temperature dependence of amorphous $\mathrm{Fe}_{80} \mathrm{~W}_{3} \mathrm{~B}_{17}$ microwire. Wolfram is known to decrease the Curie temperature of amorphous alloys [4]. Hence, magnetoelastic anisotropy decreases, too. As a result, strong and complex variation of the switching field with temperature is observed.

\section{Experimental}

The study has been performed on glass-coated amorphous microwires with nominal composition of $\mathrm{Fe}_{80} \mathrm{~W}_{3} \mathrm{~B}_{17}$, prepared by the modified Taylor-Ulitovsky technique. The diameter of the metallic core was $12.5 \mu \mathrm{m}$

*corresponding author; e-mail: peter.klein@upjs.sk and the total diameter $25 \mu \mathrm{m}$. In order to decrease the strong stresses introduced during microwire production, all samples have been annealed at $300^{\circ} \mathrm{C}$ for $1 \mathrm{~h}$ and slowly cooled down to room temperature (for $12 \mathrm{~h}$ ) to relax the stresses introduced by glass due to different thermal expansion coefficients of glass coating and metallic nucleus.

The length of the sample used for measurements varied from 1.3 to $12 \mathrm{~cm}$.

The switching field has been measured by induction method at the frequency $1000 \mathrm{~Hz}$ with maximum amplitude $1000 \mathrm{~A} / \mathrm{m}$.

More details on the experimental setup can be found elsewhere [5].

\section{Results and discussion}

Due to existence of two contributions to the domain wall potential of the closure domain wall in amorphous microwires, the switching field $H_{s w}$ of bistable microwires can be satisfactorily described by two contributions. Firstly, it is the structural relaxation contribution [5]. However, it has been shown that $\mathrm{W}$ hinders significantly the structural relaxation in amorphous FeB alloys [4]. In order to avoid this contribution completely, the measuring frequency has been set to $1000 \mathrm{~Hz}$ as this contribution is practically negligible at higher measuring frequencies [5]. On the other hand, magnetoelastic contribution of the switching field is dominant at high frequencies. It is proportional to the energy of closure domain wall [6]:

$$
H_{s w}^{\sigma} \approx \frac{\sqrt{A \lambda_{S} \sigma}}{\mu_{0} M_{S}}
$$

where $A$ is the exchange constant, $\lambda_{S}$ is the saturation magnetostriction, $\mu_{0}$ is magnetic permeability of vacuum and $\sigma$ is given by the sum of the stresses introduced during production process $\left(\sigma_{i}\right)$ together with stress applied on the microwire by the glass due to different thermal expansion coefficients of metallic nucleus and glass-coating $\left(\sigma_{a}\right)$.

The stresses $\sigma_{a}(T)$ arising from the different thermal expansion coefficient of metallic nucleus $\alpha_{m}$ and glass 
coating $\alpha_{g}$ are proportional to the temperature $T$ :

$$
\sigma_{a}(T) \approx E_{g l}\left(\alpha_{m}-\alpha_{g}\right) \Delta T,
$$

where $E_{g l}$ is Young modulus of the glass.

The temperature dependence of magnetoelastic contribution is mainly given by the temperature dependence of the stresses introduced by the glass on the metallic nucleus $\sigma_{a}$.

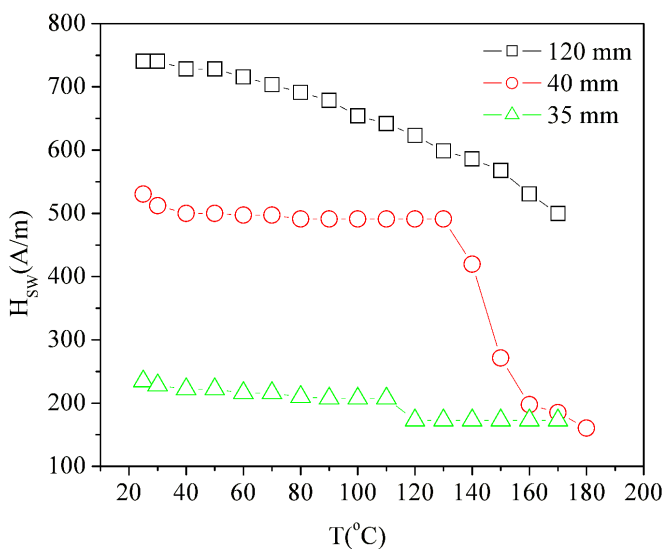

Fig. 1. Temperature dependence of the switching field $\mathrm{H}_{s w}$ for long FeWB microwires annealed at $300{ }^{\circ} \mathrm{C}$.

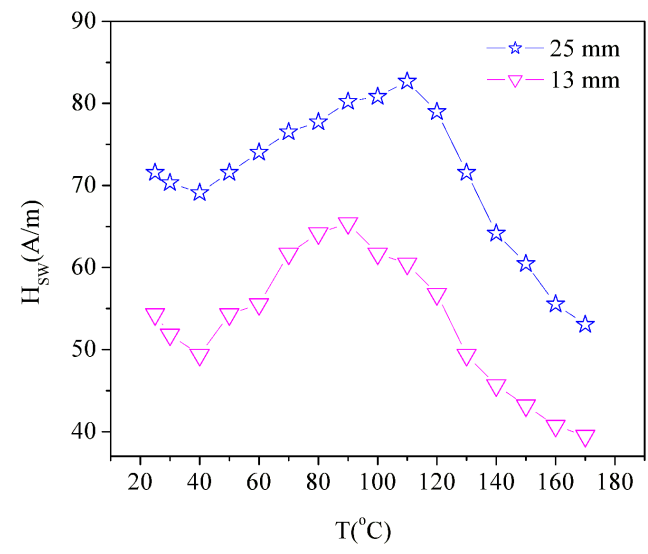

Fig. 2. Temperature dependence of the switching field $\mathrm{H}_{s w}$ for short FeWB microwires annealed at $300{ }^{\circ} \mathrm{C}$.

Temperature dependence of the switching field $H_{s w}$ for FeWB microwires with different lengths is shown in Fig. 1. As a result of strong stresses introduced during microwires production, the amplitude of the switching field is high $\sim 750 \mathrm{~A} / \mathrm{m}$ at room temperature for longest microwire $(120 \mathrm{~mm})$. As a result of reduction of the stresses applied by glass-coating, $\sigma_{a}(T)$, the switching field decreases with temperature monotonously.

The decrease of the length of microwire leads to the switching field reduction down to $540 \mathrm{~A} / \mathrm{m}$ for microwire of length of $40 \mathrm{~mm}$. The glass-coating induces high mechanical stresses on the metallic core. These stresses decrease significantly with decreasing the length of the sample. Moreover, shape anisotropy (that decreases with decreasing length of the microwire) plays also very im- portant role. On the other hand, the temperature dependence of the switching field becomes more complex, showing strong decrease above $130{ }^{\circ} \mathrm{C}$. At this temperature, the anisotropy decreases steeply as it approaches the Curie temperature (this microwire looses bistability above $180{ }^{\circ} \mathrm{C}$ ).

The effect is even more enhanced when the microwire's length decreases to $35 \mathrm{~mm}$. The switching field decreases down to $240 \mathrm{~A} / \mathrm{m}$ and its decrease shifts down to $110{ }^{\circ} \mathrm{C}$.

However, situation is more complex for very short FeWB microwires with length $25 \mathrm{~mm}$ and $13 \mathrm{~mm}$ (see Fig. 2). The switching field decreases steeply down to 72 and $55 \mathrm{~A} / \mathrm{m}$ respectively due to much smaller mechanical stresses applied by glass-coating.

Moreover, the temperature dependence of switching field becomes complex. Firstly, switching field increases with increasing temperature up to $110^{\circ} \mathrm{C}$ and $90{ }^{\circ} \mathrm{C}$ for $25 \mathrm{~mm}$ and $13 \mathrm{~mm}$ long microwires, respectively. Then, switching field decreases with increasing temperature mainly due to low value of the Curie temperature.

Such temperature dependence approves that magnetoelastic anisotropy is no more dominant. However, strong variations of the switching field could be employed for sensing the temperature.

\section{Conclusions}

In this contribution, we have studied the temperature dependence of the switching field in amorphous FeWB microwires with low Curie temperature. It is shown that decrease of the microwire's length significantly decreases magnetoelastic anisotropy that results in a complex temperature dependence of the switching field. Such strong variations can be employed for sensing the temperature in miniature applications.

\section{Acknowledgments}

This work was supported by the project NanoCEXmat Nr. ITMS 26220120019, Slovak VEGA grant. No. 1/0060/13, APVV-0027-11 and APVV-0266-10.

\section{References}

[1] M. Vázquez, H. Chiriac, A. Zhukov, L. Panina, T. Uchiyama, Phys. Stat. Sol. A 208, 493 (2011).

[2] D. Praslička, J. Blazek, M. Smelko, J. Hudak, A. Cverha, I. Mikita, R. Varga, A. Zhukov, IEEE Trans. Magn. 49, 128 (2013).

[3] N. Lupu, H. Chiriac, S. Corodeanu, G. Ababei, IEEE Trans. Magn. 47, 3791 (2011).

[4] R. Varga, P. Vojtanik, A. Lovas, J. Phys. IV France 8, Pr2-63 (1998).

[5] P. Klein, R. Varga, G.A. Badini, M. Vazquez, IEEE Trans. Magn. 46, 357 (2010).

[6] M. Vazquez, A. Hernando, J. Phys. D: Appl. Phys. 29, 939 (1996). 